\title{
WEAKLY COMPACT HOMOMORPHISMS
}

\author{
J. E. GALÉ, T. J. RANSFORD AND M. C. WHITE
}

\begin{abstract}
We study the structure of weakly compact homomorphisms between Banach algebras. In particular, it is shown that between many pairs of algebras, the only weakly compact homomorphisms are those of finite rank.
\end{abstract}

\section{INTRODUCTION}

Let $A$ and $B$ be complex Banach algebras, not necessarily unital. In this paper we investigate the structure of those homomorphisms $\theta: A \rightarrow B$ which are weakly compact, i.e. which take bounded subsets of $A$ to weakly relatively compact subsets of $B$. In particular we show that, under various assumptions on the pair $(A, B)$, the range $\theta(A)$ is necessarily finite-dimensional and semisimple, and therefore, by the Wedderburn-Artin structure theorem, isomorphic to a finite direct sum of full matrix algebras.

Our results build on previous work in the area, which has concentrated on compact endomorphisms of commutative Banach algebras [11, 12, 13], compact homomorphisms between $C^{*}$-algebras [9], and compact and weakly compact homomorphisms between uniform algebras [18]. These references also provide a fund of interesting examples of weakly compact homomorphisms, most of them actually compact (see $\S 4$ ). Such homomorphisms also arise naturally out of the factorization theory of linear operators (see $\S 3$ ), for if $\theta$ factors through a reflexive Banach space, then it is automatically weakly compact.

We conclude this introductory section by posing a converse problem.

Problem 1.1. Let $A$ and $B$ be Banach algebras. If $\theta: A \rightarrow B$ is a weakly compact homomorphism, then do there exist a reflexive Banach algebra $C$ and continuous homomorphisms $\varphi: A \rightarrow C$ and $\psi: C \rightarrow B$ such that $\theta=\psi \circ \varphi$ ?

The corresponding statement for linear maps between Banach spaces is known to be true (see e.g. [5; 15, Theorem 2.g.11]), but its proof fails to adapt to our situation because the interpolation method used does not respect Banach algebras.

\section{HOMOMORPHISMS FROM AMENABLE ALGEBRAS}

Consider the inclusion map from $C^{1}[0,1]$ to $C[0,1]$, and the endomorphism $f(z) \mapsto f(z / 2)$ of the disc algebra $A(\bar{\Delta})$. Using the Arzela-Ascoli theo-

Received by the editors March 22, 1990.

1991 Mathematics Subject Classification. Primary 46H05; Secondary 43A22, 46H20, 46L05, $47 \mathrm{~B} 10$.

The research of the first author has been partially supported by the Spanish DGICYT Project P587-0059. 
rem it is easily verified that, relative to the usual Banach-algebra norms on these spaces, both maps are compact. In each case, the compactness arises in an essential way from the differentiability properties of the domain. This suggests looking at algebras with 'no differentiability'.

The following definition is due to Johnson [10]. A Banach algebra $A$ is amenable if every continuous derivation from $A$ into a dual Banach $A$-bimodule is inner. In particular, this condition rules out the possibility of nonzero continuous point derivations on $A$, and therefore neither $C^{1}[0,1]$ nor $A(\bar{\Delta})$ is amenable. Johnson showed that if $G$ is a locally compact group, then the group algebra $L^{1}(G)$ is amenable if and only if $G$ is amenable in the classical sense (i.e. possesses an invariant mean), hence in particular whenever $G$ is compact or abelian. Other examples of amenable algebras include the uniform algebra $C(K)$ of all continuous functions on a compact space $K$, and the $C^{*}$-algebra $\mathscr{K}(H)$ of compact operators on a separable Hilbert space $H$. Moreover, if $A$ is amenable and there exists a continuous homomorphism from $A$ onto a dense subalgebra of another Banach algebra $B$, then $B$ is also amenable. Further information on amenability may be found in [4, 10 and $\S 43$ of 2].

We can now state the main theorem of this section.

Theorem 2.1. Let $A$ be an amenable Banach algebra and let $B$ be a Banach algebra all of whose irreducible representations are finite-dimensional. If $\theta$ : $A \rightarrow B$ is a weakly compact homomorphism and $\overline{\theta(A)}=B$, then $\theta(A)$ is finite-dimensional and semisimple.

The proof of Theorem 2.1 relies on the following lemma, which will also be used in $\S 3$.

Lemma 2.2. Let $A$ and $B$ be Banach algebras, and let $\theta: A \rightarrow B$ be a weakly compact homomorphism such that $\overline{\theta(A)}=B$. If I is a closed ideal in $B$ of finite codimension, and if $\theta^{-1}(I)$ has a bounded approximate identity, then $I=B p$ for some central idempotent $p$ in $B$.

Proof. First we observe that $\overline{\theta\left(\theta^{-1}(I)\right)}=I$. For, writing $J$ for the left-hand side, we certainly have $J \subset I$; also if $\tilde{\theta}: A / \theta^{-1}(I) \rightarrow B / J$ denotes the induced homomorphism and $\pi: B / J \rightarrow B / I$ is the quotient map, then $\pi \circ \tilde{\theta}$ is injective, from which it follows in turn that $\operatorname{dim}\left(A / \theta^{-1}(I)\right)<\infty$, that $\tilde{\theta}$ is surjective, that $\pi$ is injective, and finally that $J=I$, as claimed.

Now let $\left(e_{\alpha}\right)$ be a bounded approximate identity for $\theta^{-1}(I)$. Since $\theta$ is weakly compact, taking a subnet if necessary we can suppose that $\theta\left(e_{\alpha}\right)$ is weakly convergent in $B$, say to $p$. As $I$ is a norm-closed subspace, it is also weakly closed, and hence $p \in I$. Now if $a \in A$, then $\theta(a) \theta\left(e_{\alpha}\right)$ converges weakly to $\theta(a) p$. Also if $a \in \theta^{-1}(I)$, then since $\left(e_{\alpha}\right)$ is a bounded approximate identity for $I$ we have $\theta(a) \theta\left(e_{\alpha}\right)$ convergent to $\theta(a)$ in norm. Hence $\theta(a) p=$ $\theta(a)$ for all $a \in \theta^{-1}(I)$, and using the observation made at the start of the proof, it follows by density that $b p=b$ for all $b \in I$. Likewise $p b=b$ for all $b \in I$, and the result now follows.

Proof of 2.1. Since $A$ is amenable, it has a bounded approximate identity (see [4, Corollary 3.2]). Therefore we can apply Lemma 2.2 with $I=B$ to deduce that $B$ must have an identity, $e$ say. 
Denote by $\Pi_{B}$ the structure space of all primitive ideals in $B$, endowed with the hull-kernel topology. By [2, Corollary 26.5], $\Pi_{B}$ is a compact space. We now proceed to prove that it is also discrete.

Take $P \in \Pi_{B}$. Then $P$ is the kernel of an irreducible representation, so by hypothesis $\operatorname{dim}(B / P)<\infty$. It follows that $P$ is in fact a maximal ideal in $B$. Also $\theta^{-1}(P)$ has finite codimension in $A$, and so since $A$ is amenable, $\theta^{-1}(P)$ has a bounded approximate identity (see [4, Corollary 3.9]). Applying Lemma 2.2 again, we deduce that $P=B p$ for some central idempotent $p$. Clearly $e-p \notin P$. However if $Q \in \Pi_{B} \backslash\{P\}$, then $e-p \in Q$ : for otherwise, since $Q$ like $P$ is maximal, it would follow that $B(e-p)+Q=B$, which would entail

$$
P=B p=B p(e-p)+Q p=Q p \subset Q,
$$

contradicting the maximality of $P$. Thus $\bigcap_{Q \neq P} Q \not \subset P$, in other words $\{P\}$ is open in $\Pi_{B}$. Also, using the maximality of $P$ once again, we have $\operatorname{hull}(\operatorname{ker}(P))$ $=\{P\}$, so $\{P\}$ is closed in $\Pi_{B}$. This proves discreteness.

Since $\Pi_{B}$ is compact and discrete, it must be finite, say $\Pi_{B}=\left\{P_{1}, \ldots, P_{n}\right\}$. Each $P_{j}=B p_{j}$ for some central idempotent $p_{j}$, so the radical of $B$ is given by

$$
\operatorname{Rad}(B)=\bigcap_{1}^{n} P_{j}=B\left(p_{1} \cdots p_{n}\right)
$$

Since $\left(p_{1} \cdots p_{n}\right)$ is a radical idempotent, it must be zero, and hence $\operatorname{Rad}(B)=$ $\{0\}$. Therefore $B$ is isomorphic to a subalgebra of $B / P_{1} \oplus \cdots \oplus B / P_{n}$ and so is finite-dimensional. Finally as $\theta(A)$ is dense in $B$, it must in fact be equal to $B$, and so it too is finite-dimensional and semisimple.

Corollary 2.3. Let $A$ be a reflexive amenable Banach algebra such that every irreducible representation is finite-dimensional. Then $A$ is finite-dimensional and semisimple.

Proof. Apply Theorem 2.1 to the identity map on $A$.

Remark. It appears to be an open problem whether every reflexive amenable Banach algebra has to be finite-dimensional. If this were indeed true, and if the answer to Problem 1.1 were yes, then it would be possible to prove a stronger form of Theorem 2.1 in which there was no hypothesis on $B$ at all. At present, the best we can do is to verify a number of special cases.

Corollary 2.4. Let $A$ and $B$ be Banach algebras such that $A$ is amenable and at least one of $A$ and $B$ is commutative. If $\theta: A \rightarrow B$ is a weakly compact homomorphism, then $\theta(A)$ is finite-dimensional and semisimple.

Proof. We can suppose without loss of generality that $\overline{\theta(A)}=B$. Then $B$ is commutative, so all its irreducible representations are one-dimensional, and Theorem 2.1 now applies.

Corollary 2.5. Let $G$ be a compact group, let $L^{1}(G)$ be the corresponding group algebra, and let $B$ be a Banach algebra. If $\theta: L^{1}(G) \rightarrow B$ is a weakly compact homomorphism, then $\theta\left(L^{1}(G)\right)$ is finite-dimensional and semisimple.

Proof. As before, we can suppose without loss of generality that $\overline{\theta\left(L^{1}(G)\right)}=$ $B$. The algebra $L^{1}(G)$ is amenable because $G$ is compact. Also, if $\pi$ is 
an irreducible representation of $B$, then $\pi \circ \theta$ is a topologically irreducible representation of $L^{1}(G)$, so using [16, Theorem 32C and 14, p. 136, Corollary 1] it follows that $\pi \circ \theta$ is finite-rank, and hence also $\pi$. Thus the result again follows from Theorem 2.1.

We conclude this section with a few results about compact homomorphisms.

Theorem 2.6. Let $A$ be an amenable Banach algebra and let $B$ be a Banach algebra which has the bounded approximation property. If $\theta: A \rightarrow B$ is a compact homomorphism and $\overline{\theta(A)}=B$, then $\theta(A)$ is finite-dimensional and semisimple.

Proof. By [2, Theorem 43.9], since $A$ is amenable it has an approximate diagonal, i.e. a bounded net $\left(\xi_{\alpha}\right)$ in the projective tensor product $A \hat{\otimes} A$ satisfying

$$
\begin{array}{cc}
\xi_{\alpha} \cdot a-a \cdot \xi_{\alpha} \rightarrow 0 & (a \in A), \\
\mu\left(\xi_{\alpha}\right) a \rightarrow a & (a \in A) .
\end{array}
$$

Here - denotes the standard bimodule action of $A$ on $A \hat{\otimes} A$, and $\mu: A \hat{\otimes} A \rightarrow A$ is the multiplication map $\mu\left(a \otimes a^{\prime}\right)=a a^{\prime}$. Now the induced map $\theta \otimes \theta: A \hat{\otimes} A \rightarrow$ $B \hat{\otimes} B$ sends the unit ball of $A \hat{\otimes} A$ into the norm-closed convex hull of the set

$$
\left\{\theta(a) \otimes \theta\left(a^{\prime}\right):\|a\| \leq 1,\left\|a^{\prime}\right\| \leq 1\right\} .
$$

Hence, as $\theta$ is compact, so is $\theta \otimes \theta$. Therefore a subnet of $\theta \otimes \theta\left(\xi_{\alpha}\right)$ converges in $B \hat{\otimes} B$, say to $\eta$. This $\eta$ then satisfies

$$
\begin{array}{cc}
\eta \cdot b-b \cdot \eta=0 & (b \in B), \\
\mu(\eta) b=b & (b \in B) .
\end{array}
$$

By [22, Proposition 5.11], the existence of such an $\eta$, together with the bounded approximation property, implies that $B$ is finite-dimensional and semisimple. Hence so is $\theta(A)$.

From this we can deduce a variant of Corollary 2.5.

Corollary 2.7. Let $A$ be an amenable Banach *-algebra and let $B$ be a $C^{*}$ algebra. If $\theta: A \rightarrow B$ is a compact *-homomorphism, then $\theta(A)$ is finitedimensional and semisimple.

Proof. We can suppose without loss of generality that $\overline{\theta(A)}=B$. This implies that the $C^{*}$-algebra $B$ is amenable, hence nuclear, and hence that it possesses the bounded approximation property (see [3]). The result now follows from Theorem 2.6.

Corollary 2.8. Let $G$ be an amenable locally compact group and let $L^{1}(G)$ be the corresponding group algebra, equipped with the involution $f^{*}(s)=\Delta\left(s^{-1}\right) \overline{f\left(s^{-1}\right)}$.

(i) If $\theta: L^{1}(G) \rightarrow L^{1}(G)$ is a compact *-homomorphism, then $\theta\left(L^{1}(G)\right)$ is finite-dimensional and semisimple.

(ii) If $\theta: L^{1}(G) \rightarrow L^{1}(G)$ is a weakly compact *-homomorphism, then $\theta^{2}\left(L^{1}(G)\right)$ is finite-dimensional and semisimple.

Proof. (i) Let $\mathscr{B}\left(L^{2}(G)\right)$ be the $C^{*}$-algebra of bounded operators on the Hilbert space $L^{2}(G)$, and let $\pi: L^{1}(G) \rightarrow \mathscr{B}\left(L^{2}(G)\right)$ be the continuous *-homomorphism given by

$$
\pi(f)(g)=f * g \quad\left(f \in L^{1}(G), g \in L^{2}(G)\right) .
$$


Applying Corollary 2.7 to $\pi \circ \theta$, we deduce that $\pi \circ \theta\left(L^{1}(G)\right)$ is finite-dimensional and semisimple, and the result follows since $\pi$ is injective.

(ii) Since $L^{1}(G)$ has the Dunford-Pettis property, $\theta$ weakly compact implies that $\theta^{2}$ is compact (see [6, Corollary VI.8.13]). Thus (ii) follows from (i).

Remark. By making stronger use of the group structure of $G$, it is possible to extend Corollaries 2.5 and 2.8. For further details see [7].

\section{HOMOMORPHISMS FROM $C^{*}$-ALGEBRAS}

In [9], Ghahramani showed that a compact homomorphism between $C^{*}$ algebras has a finite-dimensional, semisimple range. The principal theorem of this section is a generalization of his result.

Theorem 3.1. Let $A$ be a $C^{*}$-algebra and let $B$ be a Banach algebra. If $\theta$ : $A \rightarrow B$ is a weakly compact homomorphism, then $\theta(A)$ is finite-dimensional and semisimple.

The proof proceeds via another theorem, valid for arbitrary Banach algebras, and of some interest in its own right.

An element $h$ of a unital Banach algebra $A$ is called hermitian if $\left\|e^{i t h}\right\|=1$ for all $t \in \mathbb{R}$. It can be shown that the set of all hermitian elements of $A$ forms a closed real subspace of $A$. For further details see [2, $\S 10$ and $\S 38]$.

Theorem 3.2. Let $A$ and $B$ be unital Banach algebras, and let $H$ be the set of hermitian elements of $A$. If $\theta: A \rightarrow B$ is a weakly compact unital homomorphism, then there exists an integer $N \geq 1$ such that every element of $\overline{\theta(H+i H)}$ is algebraic over $\mathbb{C}$, of degree at most $N$.

We shall need a simple analytic lemma.

Lemma 3.3. Let $D$ be a subdomain of $\mathbb{C}$, let $X$ be a complex Banach space, and let $f_{0}, \ldots, f_{N}: D \rightarrow X$ be holomorphic functions. Define $E$ to be the set of $\lambda \in D$ for which $\left\{f_{0}(\lambda), \ldots, f_{N}(\lambda)\right\}$ is linearly dependent. Then either $E=D$ or $E$ contains only isolated points.

Proof. Suppose that $E \neq D$, say $\lambda_{0} \in D \backslash E$. Using the Hahn-Banach theorem, we may choose $\gamma_{0}, \ldots, \gamma_{N} \in X^{*}$ such that $\gamma_{j}\left(f_{k}\left(\lambda_{0}\right)\right)=\delta_{j k}$. Then the determinant

$$
\left|\begin{array}{ccc}
\gamma_{0}\left(f_{0}(\lambda)\right) & \cdots & \gamma_{0}\left(f_{N}(\lambda)\right) \\
\vdots & & \vdots \\
\gamma_{N}\left(f_{0}(\lambda)\right) & \cdots & \gamma_{N}\left(f_{N}(\lambda)\right)
\end{array}\right|
$$

is a holomorphic function of $\lambda$ which is zero on $E$, but not identically zero on $D$ (for it equals 1 at $\lambda_{0}$ ). Therefore $E$ contains only isolated points.

Proof of 3.2. We first show that if $h \in H$ then $\theta(h)$ is algebraic. Fix $h \in H$, and define $\varphi: l^{1}(\mathbb{Z}) \rightarrow A$ by

$$
\varphi\left(\left(a_{n}\right)\right)=\sum_{-\infty}^{\infty} a_{n} e^{i n h} \quad\left(\left(a_{n}\right) \in l^{1}(\mathbb{Z})\right) .
$$

Since $\left\|e^{i n h}\right\|=1$ for all $n$, this is a well-defined, continuous homomorphism. Therefore $\theta \circ \varphi: l^{1}(\mathbb{Z}) \rightarrow B$ is a weakly compact homomorphism, and so by 
Corollary 2.4 it has finite-dimensional range. This implies that $e^{i \theta(h)}$ is algebraic, say $p\left(e^{i \theta(h)}\right)=0$. If we factor $p\left(e^{i z}\right)$ as $q(z) r(z)$, where $q$ is a polynomial and $r$ is nonzero on the spectrum $\sigma_{B}(\theta(h))$, it follows that $q(\theta(h))=0$. Thus $\theta(h)$ is algebraic, as claimed.

Now for $n \geq 1$, define

$$
H_{n}=\left\{h \in H: \exists \alpha_{0}, \ldots, \alpha_{n} \in \mathbb{C}, \sum_{0}^{n}\left|\alpha_{j}\right|=1, \sum_{0}^{n} \alpha_{j} \theta(h)^{j}=0\right\} .
$$

Then each $H_{n}$ is closed in $H$, and by the first part of the proof $\bigcup_{n} H_{n}=H$. Since $H$ is complete, we can apply Baire's theorem to deduce that int ${ }_{H}\left(H_{N}\right) \neq$ $\varnothing$ for some $N$. Fix $h_{0} \in \operatorname{int}_{H}\left(H_{N}\right)$. Given $h, k \in H$, define $f_{0}, \ldots, f_{N}$ : $\mathbb{C} \rightarrow B$ by

$$
f_{j}(\lambda)=\theta\left(h_{0}+\lambda k+\lambda^{2}\left(h_{0}-h\right)\right)^{j} \quad(\lambda \in \mathbb{C}, 0 \leq j \leq N) .
$$

If $\lambda$ is real and sufficiently small, then $h_{0}+\lambda k+\lambda^{2}\left(h_{0}-h\right) \in H_{N}$, so $\left\{f_{0}(\lambda), \ldots\right.$, $\left.f_{N}(\lambda)\right\}$ is linearly dependent. By Lemma 3.3 it follows that $\left\{f_{0}(\lambda), \ldots, f_{N}(\lambda)\right\}$ is linearly dependent for all $\lambda \in \mathbb{C}$, in particular for $\lambda=i$. Translating this back again, we deduce that $\theta(h+i k)$ is algebraic of degree at most $N$. The set of all elements with this property is closed in $B$, hence it contains $\overline{\theta(H+i H)}$.

Remark. The complex vector space $H+i H$ in Theorem 3.2 is not in general a subalgebra of $A$, though it does form a Lie subalgebra (see [2, Lemma 38.2]). If $J$ is the subalgebra generated by $H+i H$, is it true that every element of $\overline{\theta(J)}$ is necessarily algebraic?

Proof of 3.1. Adjoining identities if necessary, we can suppose that $A, B$, and $\theta$ are unital, and that $\overline{\theta(A)}=B$. Then since $A$ is a $C^{*}$-algebra, every selfadjoint element is hermitian, and so in the notation of Theorem 3.2, $H+$ $i H=A$. Hence there exists $N \geq 1$ such that every element of $B$ is algebraic of degree at most $N$. Using standard representation theory (see e.g. [1, Lemma 3.2.1]), it follows that the $\operatorname{radical} \operatorname{Rad}(B)$ has finite codimension in $B$. Since $\left.\theta^{-1} \operatorname{Rad}(B)\right)$ is a closed ideal in a $C^{*}$-algebra, it has a bounded approximate identity (see [2, Lemma 39.14]). So Lemma 2.2 applies, and we deduce that $\operatorname{Rad}(B)=B p$ for some central idempotent $p$. As in the proof of Theorem 2.1, this implies that $\operatorname{Rad}(B)=\{0\}$. Thus $B$ is finite-dimensional and semisimple, and hence so is $\theta(A)$.

To round off this section, we give two applications of Theorem 3.1. The first of these is already known, though previous proofs (see e.g. [21]) are not altogether easy.

Corollary 3.4. A reflexive $C^{*}$-algebra is finite-dimensional.

Proof. Apply Theorem 3.1 to the identity map.

Remark. The result stated in Corollary 3.4 can be used as the basis for a second proof of Theorem 3.1. This proof was discovered independently by M. Mathieu, G. A. Willis, and one of the authors, and will appear in [17].

Our second application exploits the link, mentioned in $\S 1$, with the factorization theory of linear operators. A Banach space $X$ has cotype 2 if there exists 
a constant $\kappa$ such that whenever $n \geq 1$ and $x_{1}, \ldots, x_{n} \in X$, then

$$
\sum_{1}^{n}\left\|x_{j}\right\|^{2} \leq \kappa^{2} \int_{0}^{1}\left\|\sum_{1}^{n} r_{j}(t) x_{j}\right\|^{2} d t,
$$

where $r_{1}, \ldots, r_{n}$ denote the first $n$ Rademacher functions on $[0,1]$. Examples of cotype 2 spaces include all $L^{p}$-spaces for $1 \leq p \leq 2$, the dual of any $C^{*}$-algebra, and more generally, any 2 -uniformly PL-convex space. For more details see $[15,19]$.

Corollary 3.5. Let $A$ be a $C^{*}$-algebra and let $B$ be a Banach algebra of cotype 2. If $\theta: A \rightarrow B$ is a continuous homomorphism, then $\theta(A)$ is finite-dimensional and semisimple.

Proof. By Pisier's noncommutative version of Grothendieck's theorem [20, Theorem 3.1], every continuous linear map from a $C^{*}$-algebra to a Banach space of cotype 2 factors through a Hilbert space, and so in particular is weakly compact. The result therefore follows from Theorem 3.1.

Remark. The results of this section also have analogues for Jordan-Banach algebras. Details will appear in [8].

\section{HOMOMORPHISMS FROM COMMUTATIVE ALGEBRAS}

If $A$ is a commutative unital Banach algebra, we denote by $\Phi_{A}$ the character space of $A$. The dual $\theta^{*}$ of a unital homomorphism $\theta$ from $A$ to another commutative Banach algebra $B$ then maps $\Phi_{B}$ into $\Phi_{A}$.

Our basic result is the following simple theorem.

Theorem 4.1. Let $A$ and $B$ be commutative unital Banach algebras, and let $\left(U_{\alpha}\right)$ be a partition of $\Phi_{A}$ into $\sigma\left(A^{*}, A^{* *}\right)$-open subsets. If $\theta: A \rightarrow B$ is a weakly compact unital homomorphism, then $\theta^{*}\left(\Phi_{B}\right)$ meets only finitely many $U_{\alpha}$.

Proof. Let $\theta^{* *}: A^{* *} \rightarrow B^{* *}$ be the second dual of $\theta$. Since $\theta$ is weakly compact, $\theta^{* *}$ actually maps $A^{* *}$ into $B$ (see [6, Theorem VI.4.2]). From this it follows easily that $\theta^{*}$ is a continuous map with respect to the topologies $\sigma\left(B^{*}, B\right)$ and $\sigma\left(A^{*}, A^{* *}\right)$. Hence $\left(\left(\theta^{*}\right)^{-1}\left(U_{\alpha}\right)\right)$ is a partition of $\Phi_{B}$ into $\sigma\left(B^{*}, B\right)$-open sets, and since $\Phi_{B}$ is $\sigma\left(B^{*}, B\right)$-compact, only finitely many of these sets can be nonempty.

As Theorem 4.1 is rather abstract, we will illustrate it with a pair of special cases. The first is a result of Ohno and Wada [18, Theorem 1.1(c)].

Corollary 4.2. Let $A$ and $B$ be uniform algebras. If $\theta: A \rightarrow B$ is a weakly compact unital homomorphism, then $\theta^{*}\left(\Phi_{B}\right)$ meets only finitely many Gleason parts of $A$.

Proof. The Gleason parts of $A$ are $\sigma\left(A^{*}, A^{* *}\right)$-open subsets of $\Phi_{A}$ (this is essentially proved in [18]). Thus the result follows from Theorem 4.1 .

If $A$ is a uniform algebra, then every peak point in $\Phi_{A}$ is $\sigma\left(A^{*}, A^{* *}\right)$ isolated. More generally, for any commutative Banach algebra $A$, a point of $\Phi_{A}$ is $\sigma\left(A^{*}, A^{* *}\right)$-isolated whenever the corresponding maximal ideal has a bounded approximate identity. Thus the next result is closely related to Theorem 2.1. 
Corollary 4.3. Let $A$ and $B$ be commutative unital Banach algebras such that $\Phi_{A}$ is $\sigma\left(A^{*}, A^{* *}\right)$-discrete and $B$ is semisimple. If $\theta: A \rightarrow B$ is a weakly compact homomorphism then $\theta(A)$ is finite-dimensional and semisimple.

Proof. We can suppose without loss of generality that $\overline{\theta(A)}=B$, so that $\theta$ is unital. By Theorem $4.1 \theta^{*}\left(\Phi_{B}\right)$ is finite, and hence so also is $\Phi_{B}$. As $B$ is semisimple, it must therefore be finite-dimensional, and thus so is $\theta(A)$.

As remarked in the introduction, most of the standard examples of infiniterank weakly compact homomorphisms are actually compact. This is at least partly explained by our final result, which was inspired by [18, Theorem 1.2].

Let us say that a commutative unital Banach algebra $A$ has $\sigma\left(A^{*}, A^{* *}\right)$ holomorphic structure if there exists a partition of $\Phi_{A}$ into $\sigma\left(A^{*}, A^{* *}\right)$-open sets $\left(U_{\alpha}\right)$, such that for each $\alpha$, either $U_{\alpha}$ is finite, or there exist $g_{1}, \ldots, g_{n} \in A$ with the following properties:

(i) the map $\hat{\mathbf{g}}$ defined by

$$
\hat{\mathbf{g}}(\chi)=\left(\chi\left(g_{1}\right), \ldots, \chi\left(g_{n}\right)\right) \quad\left(\chi \in U_{\alpha}\right)
$$

is a homeomorphism of $U_{\alpha}$ onto an open subset $V_{\alpha}$ of $\mathbb{C}^{n}$, and

(ii) for each $a \in A$, the function $\hat{a} \circ \hat{\mathbf{g}}^{-1}$ is holomorphic on $V_{\alpha}$. (The integer $n$ may depend on $\alpha$; the case when $U_{\alpha}$ is finite may be thought of as the degenerate case $n=0$.) Examples of such algebras include $l^{1}\left(\mathbb{Z}^{+}\right)$, the unitization of $L^{1}\left(\mathbb{R}^{+}\right)$, the disc algebra $A(\bar{\Delta})$, and their multi-variable counterparts.

Theorem 4.4. Let $A$ and $B$ be commutative unital Banach algebras such that $A$ has $\sigma\left(A^{*}, A^{* *}\right)$-holomorphic structure and $B$ is semisimple. If $\theta: A \rightarrow B$ is a weakly compact homomorphism then $\theta$ is compact.

Proof. We can suppose without loss of generality that $\overline{\theta(A)}=B$, so that $\theta$ is unital. Let $\left(U_{\alpha}\right)$ be a $\sigma\left(A^{*}, A^{* *}\right)$-open partition of $\Phi_{A}$ satisfying the condition above. By Theorem 4.1, $\theta^{*}\left(\Phi_{B}\right)$ meets only finitely many $U_{\alpha}$, say $U_{1}, \ldots, U_{m}$, and $\left(\left(\theta^{*}\right)^{-1}\left(U_{j}\right)\right)$ then provides a $\sigma\left(B^{*}, B\right)$-open partition of $\Phi_{B}$. By the Shilov idempotent theorem [2, Theorem 21.5], there exist idempotents $p_{1}, \ldots, p_{m} \in B$ such that for each $j$

$$
\chi\left(p_{j}\right)= \begin{cases}1, & \theta^{*}(\chi) \in U_{j}, \\ 0, & \theta^{*}(\chi) \notin U_{j} .\end{cases}
$$

Now $B p_{j}$ is a semisimple commutative Banach algebra, which has identity $p_{j}$ and character space $\left(\theta^{*}\right)^{-1}\left(U_{j}\right)$. Define a continuous homomorphism $\theta_{j}: A \rightarrow$ $B p_{j}$ by

$$
\theta_{j}(a)=\theta(a) p_{j} \quad(a \in A) .
$$

Since $\sum_{1}^{m} p_{j}$ is the identity on $B$, it follows that $\theta=\sum_{1}^{m} \theta_{j}$, and so to prove that $\theta$ is compact, it suffices to do so for each $\theta_{j}$. In other words, we may assume without loss of generality that the original $\theta$ has the property that $\theta^{*}\left(\Phi_{B}\right) \subset U$, where $U$ is a single $U_{\alpha}$.

There are two cases to consider. First suppose that $U$ is finite. Then $B$ has a finite character space, and so because it is semisimple it must be finitedimensional. So in this case $\theta$ is certainly compact.

Now suppose that we are in the other case, and let $g_{1}, \ldots, g_{n}$ be elements of $A$ with the properties (i) and (ii) stated above. Let $V$ be the open subset $\hat{\mathbf{g}}(U)$ 
of $\mathbb{C}^{n}$, and denote by $\mathscr{O}(V)$ the Fréchet algebra of all functions holomorphic on $V$. Then we can define a continuous homomorphism $\varphi: A \rightarrow \mathscr{O}(V)$ by

$$
\varphi(a)=\hat{a} \circ \hat{\mathbf{g}}^{-1} \quad(a \in A) .
$$

Also the joint spectrum $\sigma_{B}\left(\theta\left(g_{1}\right), \ldots, \theta\left(g_{n}\right)\right)$, which is just $\hat{\mathbf{g}}\left(\theta^{*}\left(\boldsymbol{\Phi}_{B}\right)\right)$, is contained in $V$, so we can use the holomorphic functional calculus (see $[2, \S 20]$ ) to define a continuous homomorphism $\psi: \mathscr{O}(V) \rightarrow B$ by

$$
\psi(f)=f\left(\theta\left(g_{1}\right), \ldots, \theta\left(g_{n}\right)\right) \quad(f \in \mathscr{O}(V)) .
$$

Then given $a \in A$ and $\chi \in \Phi_{B}$, we have

$$
\begin{aligned}
\chi(\psi \circ \varphi(a)) & =\chi\left(\hat{a} \circ \hat{\mathbf{g}}^{-1}\left(\theta\left(g_{1}\right), \ldots, \theta\left(g_{n}\right)\right)\right) \\
& =\hat{a} \circ \hat{\mathbf{g}}^{-1}\left(\chi\left(\theta\left(g_{1}\right)\right), \ldots, \chi\left(\theta\left(g_{n}\right)\right)\right) \\
& =\hat{a}\left(\theta^{*}(\chi)\right)=\chi(\theta(a)) .
\end{aligned}
$$

As $B$ is semisimple, it follows that $\theta=\psi \circ \varphi$. Thus $\theta$ factors through $\mathscr{O}(V)$, and this implies that it must be compact.

Remark. For certain algebras $A$, it is possible to give an explicit description of the structure of $\theta$, and thereby obtain an alternative proof of Theorem 4.4 in these cases. For further details see [7].

\section{ACKNOWLEDGMENTS}

This paper benefitted from the helpful comments of many people; in particular we would like to thank H. G. Dales, D. J. H. Garling, R. G. Haydon, N. J. Kalton, and G. A. Willis.

\section{REFERENCES}

1. B. Aupetit, Propriétés spectrales des algèbres de Banach, Lecture Notes in Math., vol. 735, Springer, Berlin, 1979.

2. F. F. Bonsall and J. Duncan, Complete normed algebras, Springer, Berlin, 1973.

3. M. D. Choi and E. G. Effros, Nuclear $C^{*}$-algebras and the approximation property, Amer. J. Math. 100 (1978), 61-80.

4. P. C. Curtis and R. J. Loy, The structure of amenable Banach algebras, J. London Math. Soc. (2) 40 (1989), 89-104.

5. W. J. Davis, T. Figiel, W. B. Johnson and A. Pełczyński, Factoring weakly compact operators, J. Funct. Anal. 17 (1974), 311-327.

6. N. Dunford and J. T. Schwartz, Linear operators. I, Interscience, New York, 1958.

7. J. E. Galé, Weakly compact homomorphisms and semigroups in Banach algebras, J. London Math. Soc. (to appear).

8. W Weakly compact homomorphisms between Jordan-Banach algebras, (to appear).

9. F. Ghahramani, Compact homomorphisms of $C^{*}$-algebras, Proc. Amer. Math. Soc. 103 (1988), 458-461.

10. B. E. Johnson, Cohomology in Banach algebras, Mem. Amer. Math. Soc. No. 127 (1972).

11. H. Kamowitz, Compact endomorphisms of Banach algebras, Pacific J. Math. 89 (1980), 313-325.

12. H. Kamowitz and D. Wortman, Endomorphisms of an extremal algebra, Proc. Amer. Math. Soc. 104 (1988), 852-858.

13. H. Kamowitz, S. Scheinberg and D. Wortman, Compact endomorphisms of Banach algebras. II, Proc. Amer. Math. Soc. 107 (1989), 417-422. 
14. A. A. Kirillov, Elements of the theory of representations, Springer, Berlin, 1976.

15. J. Lindenstrauss and L. Tzafriri, Classical Banach spaces. II, Springer, Berlin, 1979.

16. L. H. Loomis, An introduction to abstract harmonic analysis, Van Nostrand, Princeton, N.J., 1953.

17. M. Mathieu, Weakly compact homomorphisms from $C^{*}$-algebras are of finite rank, Proc. Amer. Math. Soc. 107 (1989), 761-762.

18. S. Ohno and J. Wada, Compact homomorphisms on function algebras, Tokyo J. Math. 4 (1981), 105-112.

19. G. Pisier, Factorization of linear operators and geometry of Banach spaces, CBMS Regional Conf. Ser. in Math., no. 60, Amer. Math. Soc., Providence, R.I., 1986.

20. _ Factorization of operators through $L_{p \infty}$ or $L_{p 1}$ and non-commutative generalizations, Math. Ann. 276 (1986), 105-136.

21. S. Sakai, Weakly compact operators on operator algebras, Pacific J. Math. 14 (1964), 659664.

22. J. L. Taylor, Homology and cohomology for topological algebras, Adv. in Math. 9 (1972), 137-182.

Departamento de Matemáticas, Universidad de Zaragoza, 50009 Zaragoza, Spain

E-mail address, J. E. Galé: gale@cc.unizar.es

D.P.M.M.S., 16 Mill Lane, Cambridge CB2 1SB, England

Current address: T. J. Ransford: D.P.M.M.S., 16 Mill Lane, Cambridge CB 21SB, England

E-mail address, T. J. Ransford: TJR11@UK.AC.CAM.PHX

Current address: M. C. White: School of Mathematics, University of Newcastle-upon-Tyne, Newcastle-upon-Tyne NE1 7RU, England

E-mail address, M. C. White: Michael.White@uk.ac.newcastle 